\title{
PSMA-Targeted Radionuclide Therapy of Metastatic Castration-Resistant Prostate Cancer with ${ }^{177}$ Lu-Labeled PSMA-617
}

\author{
Clemens Kratochwil ${ }^{1}$, Frederik L. Giesel ${ }^{1,2}$, Melsa Stefanova ${ }^{1}$, Martina Benešová ${ }^{3}$, Marcus Bronzel ${ }^{4}$, \\ Ali Afshar-Oromieh ${ }^{1,2}$, Walter Mier ${ }^{1}$, Matthias Eder ${ }^{3}$, Klaus Kopka ${ }^{3}$, and Uwe Haberkorn ${ }^{1,2}$ \\ ${ }^{1}$ Department of Nuclear Medicine, University Hospital Heidelberg, Heidelberg, Germany; ${ }^{2}$ Clinical Cooperation Unit Nuclear \\ Medicine, German Cancer Research Center (dkfz), Heidelberg, Germany; ${ }^{3}$ Division of Radiopharmaceutical Chemistry, German \\ Cancer Research Center (dkfz), Heidelberg, Germany; and ${ }^{4} A B X-C R O$, Dresden, Germany
}

\begin{abstract}
Prostate-specific membrane antigen (PSMA) is an excellent target for radionuclide therapy of metastasized castration-resistant prostate cancer (mCRPC). Besides high affinity and long tumor retention, the DOTA-conjugated ligand PSMA-617 has low kidney uptake, making it an excellent choice for therapeutic application. We retrospectively report our experience with ${ }^{177} \mathrm{Lu}$-PSMA-617-targeted radionuclide therapy in a case series of mCRPC patients resistant to other treatments. Methods: Patients with PSMA-positive tumor phenotypes were selected by molecular imaging. Thirty patients received 1-3 cycles of ${ }^{177}$ Lu-PSMA-617. During therapy, pharmacokinetics and radiation dosimetry were evaluated. Blood cell count was checked every 2 wk after the first and every 4 wk after succeeding cycles. Prostate-specific antigen (PSA) was determined every 4 wk. Radiologic restaging was performed after 3 cycles. Results: Twenty-one of 30 patients had a PSA response; in 13 of 30 the PSA decreased more than $50 \%$. After 3 cycles, 8 of 11 patients achieved a sustained PSA response $(>50 \%)$ for over $24 \mathrm{wk}$, which also correlated with radiologic response (decreased lesion number and size). Normally, acute hematotoxicity was mild. Diffuse bone marrow involvement was a risk factor for higher grade myelosuppression but could be identified by PSMA imaging in advance. Xerostomia, nausea, and fatigue occurred sporadically $(<10 \%)$. Clearance of non-tumor-bound tracer was predominantly renal and widely completed by $48 \mathrm{~h}$. Safety dosimetry revealed kidney doses of approximately $0.75 \mathrm{~Gy} / \mathrm{GBq}$, red marrow doses of $0.03 \mathrm{~Gy} / \mathrm{GBq}$, and salivary gland doses of $1.4 \mathrm{~Gy} / \mathrm{GBq}$, irrespective of tumor burden and consistent on subsequent cycles. Mean tumor-absorbed dose ranged from 6 to 22 Gy/GBq during cycle 1 . Conclusion: ${ }^{177}$ Lu-PSMA-617 is a promising new option for therapy of mCRPC and deserves more attention in larger prospective trials.
\end{abstract}

Key Words: PSMA; ${ }^{177} \mathrm{Lu}$; castration-resistant prostate cancer; radionuclide therapy; pharmacokinetics; dosimetry

J Nucl Med 2016; 57:1170-1176

DOI: 10.2967/jnumed.115.171397

Received Dec. 16, 2015; revision accepted Feb. 18, 2016.

For correspondence or reprints contact: Clemens Kratochwil, Department of Nuclear Medicine, University of Heidelberg, Im NeuenheimerFeld 400, 69120 Heidelberg, Germany.

E-mail: clemens.kratochwil@med.uni-heidelberg.de

Published online Mar. 16, 2016.

COPYRIGHT (c) 2016 by the Society of Nuclear Medicine and Molecular Imaging, Inc.
D castration-resistant prostate cancer (mCRPC) remains a lethal disease and additional treatment options are still needed.

Prostate-specific membrane antigen (PSMA) is a promising target for directing new therapies. It is found in most prostate cancers (1), and its overexpression correlates with traditional adverse prognostic factors (2). Binding of a ligand leads to internalization via clathrincoated pits (3) and prolonged retention in the cell. PSMA antibodyauristatin conjugates have been considered 1 option (4), but they face the inherent resistance of $\mathrm{mCRPC}$ against most (excepting taxanes) conventional chemotherapies. In contrast, prostate cancer is usually radiosensitive. Radiotherapy is a standard treatment for localized prostate cancer, for palliative management of mCRPC, and even radiopharmaceuticals targeting the surrounding bone matrix instead of the tumor itself can improve survival (5). Therefore, it seems more promising that a radioactive PSMA ligand, which is directly internalized into tumor cells, will be effective in delivering high doses for systemic endoradiotherapy. A phase 2 study using the radiolabeled antibody ${ }^{177}$ Lu-J591 already demonstrated moderate antitumor effects $(6)$, but the slow diffusion of antibodies into solid lesions and hematotoxicity caused by a long circulation time in blood are limitations $(7,8)$. Because of faster kinetics, the PSMA-targeted smallmolecule MIP-1095, when labeled with ${ }^{131} \mathrm{I}$, demonstrated outcomes that were superior to the antibody approach with prostate-specific antigen (PSA) responses in 17 of 28 patients (9). Unfortunately, the coemission of high-energy photons from ${ }^{131} \mathrm{I}$ requires elaborate radiation protection. Unlike ${ }^{131} \mathrm{I},{ }^{177} \mathrm{Lu}$ is a more pure $\beta$-particle emitter and preferable for clinical routine. The DOTA-conjugated

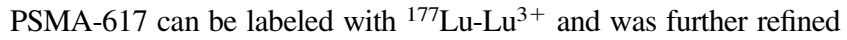
in tumor-targeting with low nanomolar affinity in the range of $0.37 \mathrm{nM}$ (NAALADase assay) and $2.34 \mathrm{nM}$ (equilibrium dissociation constant on $\mathrm{LNCaP}$ ) and highly efficient internalization with approximately $75 \%$ of the total cell associated activity internalized after $3 \mathrm{~h}$ of incubation on LNCaP (10-12).

Here, we report our first clinical experience with ${ }^{177} \mathrm{Lu}-\mathrm{PSMA}-$ 617 in patients with advanced mCRPC resistant to or with contraindications to other conventional therapies and PSMA-positive tumor phenotypes as demonstrated by molecular imaging using structurally related diagnostic analogs (Fig. 1). All used PSMA ligands share the Glu-urea motif for binding to the proteolytic domain and a lipophilic chelate or linker region to interact with the hydrophobic accessory pocket proposed by Bařinka et al. (13). 


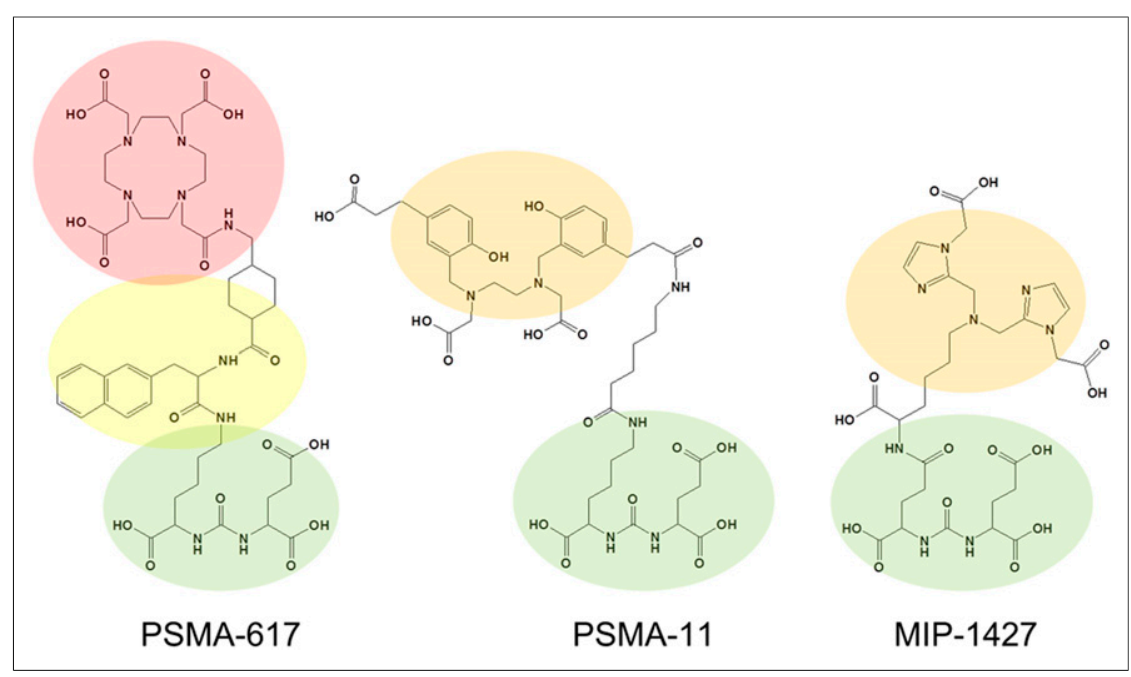

FIGURE 1. PSMA equals enzyme glutamate carboxypeptidase II. Its proteolytic domain can be targeted with Glu-urea motif (green). Hydrophobic pocket accessory to proteolytic domain adversely interacts with highly polar chelates such as DOTA (red) but favors more lipophilic chelates (orange) such as CIM (MIP-1427) for labeling with ${ }^{99 m}$ Tc or HBED-CC (PSMA-11) for labeling with ${ }^{68} \mathrm{Ga}$. In PSMA-617, aromatic linker (yellow) exploits lipophilic accessory pocket to keep more universal DOTA-chelate remote to Glu-urea binding site.

\section{MATERIALS AND METHODS}

\section{Patients}

${ }^{177}$ Lu-PSMA-617 was offered as surrogate therapy in accordance with the updated Declaration of Helsinki, paragraph-37 "Unproven Interventions in Clinical Practice," and in accordance with German regulations for "compassionate use," which includes priority of all approved treatments (without contraindications) and confirmation of the indication by both a nuclear medicine physician and an external expert in urology or oncology. In brief, all 30 patients were refractory to luteinizing hormone-releasing hormone analogs and antiandrogens (Table 1). Twenty-three patients underwent prior treatment with abiraterone or enzalutamide; 11 of them had received both. Fourteen patients were refractory to docetaxel; 4 had subsequently also been treated with cabazitaxel and 4 with estramustine. Six patients were pretreated with ${ }^{223} \mathrm{Ra}$. In contrast to a formal clinical trial, no systematic patient selection was performed, except all patients had to present with a PSMA-positive tumor phenotype based on PSMA imaging. All patients were informed about the experimental nature of this therapy and gave written informed consent. The institutional review board approved this retrospective study.

\section{Imaging-Based Patient Stratification}

PSMA imaging was performed less than $4 \mathrm{wk}$ before the first treatment cycle. Two different kinds of PSMA imaging were used before treatment.

Patients with a public health care provider (only reimbursement for scintigraphy) underwent planar scanning and dual-bed-position SPECT/ CT (Infinity; GE Healthcare) covering the thorax/abdomen/pelvis $3 \mathrm{~h}$ after intravenous injection of 500-700 MBq of ${ }^{99 \mathrm{~m} T c-M I P 1427 ~(50 ~ n m o l ~}$ ligand). The precursor was produced in-house as previously described (14) and labeled according to the protocol described with minor modifications; in short the deprotected precursor was radiolabeled with the tricarbonyl method using the CRS Isolink kit (PSI). The intensity of tumor uptake was scored visually.

Patients with a commercial health care provider and reimbursement for PET/CT underwent PSMA PET/CT. This imaging was performed either in our department on a Biograph 6 PET/CT scanner (Siemens) at $1 \mathrm{~h}$ after injection of $150 \mathrm{MBq} \pm 20 \%$ ( $2 \mathrm{nmol}$ ligand) of ${ }^{68} \mathrm{Ga}$-PSMA-11
(15) or at outside PET centers before the patients were scheduled to receive therapy in our department. PSMA PET scans were quantified by measuring SUV max $_{\text {ax }}$ for the hottest bone, soft-tissue, and lymph node metastasis (as prospectively defined index lesions), respectively.

\section{${ }^{177}$ Lu Labeling of PSMA-617}

The precursor PSMA-617 was synthesized as described previously (10) or was obtained from $\mathrm{ABX}$ advanced biochemical compounds and dissolved with dimethyl sulfoxide to obtain a $10 \mathrm{mM}$ solution. Two microliters (20 nmol) of this solution were used per 1 $\mathrm{GBq}$ of ${ }^{177} \mathrm{Lu}^{-\mathrm{LuCl}_{3}}$ (NEZ307D [Perkin Elmer], $0.04 \mathrm{M} \mathrm{HCl}$ ) mixed with $1.25 \mu \mathrm{L}$ of $20 \%$ ascorbic acid and $100 \mu \mathrm{L}$ of $0.4 \mathrm{M}$ sodium acetate buffer ( $\mathrm{pH} 5$; adjusted with acetic acid) and injected directly into the ${ }^{177} \mathrm{Lu}-$ $\mathrm{LuCl}_{3}$ delivery vial. After being heated to $95^{\circ} \mathrm{C}$ for $10 \mathrm{~min}$, a quality check per reversedphase high-performance liquid chromatography and instant thin-layer chromatography was performed, and the final product was diluted in $2 \mathrm{~mL}$ of $0.9 \% \mathrm{NaCl}$.

\section{Pharmacokinetics and Dosimetry}

Thorough descriptions of the methods used for evaluation of pharmacokinetics and dosimetry are provided in the supplemental materials (available at http://jnm.snmjournals.org).

\section{Treatment Regime and Follow-up}

According to German radiation protection laws, the patients were treated as in-patients on the nuclear medicine ward until $48 \mathrm{~h}$ after injection. Clinical examination was performed before and $1 \mathrm{~d}$ after therapy. Patients received intravenous hydration $(2,000 \mathrm{~mL}$ of $0.9 \%$ $\mathrm{NaCl}$; flow, $333 \mathrm{~mL} / \mathrm{h}$ ) starting $30 \mathrm{~min}$ before therapy. The therapy solution was administered with a slow (30-60 s) freehand injection through a $0.20-\mu \mathrm{m}$ sterile filter with low protein binding (Filtropur $\mathrm{S}$ 0.2 ; Sarstedt). Our initial treatment regime was based on 3.7-4.0 GBq per cycle repeated every $2 \mathrm{mo}$, which was derived from data with ${ }^{131} \mathrm{I}$ MIP1095 (9). Once first ligand-specific dosimetry data became available for ${ }^{177} \mathrm{Lu}-\mathrm{PSMA}-617$, the dose was increased to $6 \mathrm{GBq}$ per cycle. An overview of the administered activities is provided in Table 1 . After the first cycle, blood cell count was done every $2 \mathrm{wk}$, and during the succeeding cycles at least every $4 \mathrm{wk}$. Serum creatinine, blood-urea-nitrogen, liver enzymes, and PSA were checked every $4 \mathrm{wk}$. Baseline and followup values of laboratory tests were classified into toxicity gradings using the Common Terminology Criteria for Adverse Events 3.0 (16). After 3 cycles, imaging-based restaging was performed with either ${ }^{68} \mathrm{Ga}-$ PSMA11 PET/CT or ${ }^{99 m}$ Tc-MIP1427 SPECT/CT as available baseline.

\section{RESULTS}

\section{Pharmacokinetics}

The initial volume of distribution $1 \mathrm{~h}$ after injection was $22 \pm 12 \mathrm{~L}$, which approximates extracellular body water (17). Comparison of full-blood samples and serum revealed that there was neither a relevant passive diffusion of PSMA-617 into cellular blood components nor absorption at their surface. Blood clearance could be fitted biexponentially with half-lives of 4 and 95 h (Supplemental Fig. 1A), interpretable as fast clearance from extracellular body water and a slow clearance averaged from organs with specific uptake 
TABLE 1

Patient Characteristics

\begin{tabular}{|c|c|c|c|c|c|c|c|c|c|c|c|}
\hline $\begin{array}{c}\text { Patient } \\
\text { no. }\end{array}$ & Age (y) & GS & OP & $\mathrm{RTx}$ & CRPC & Abirat & Enza & ${ }^{223} \mathrm{Ra}$ & CTx & Cycles (GBq) & $\begin{array}{c}\text { Visceral } \\
\text { metastases }\end{array}$ \\
\hline 1 & 68 & 7 & 1 & B & 1 & 0 & 0 & 0 & $\mathrm{D}$ & $6 / 6 / 6$ & Lung \\
\hline 2 & 71 & 4 & 0 & L/B & 1 & 1 & 1 & 0 & $\mathrm{D} / \mathrm{C}$ & $4 / 4 / 4$ & Liver \\
\hline 3 & 75 & 9 & 1 & B & 1 & 0 & 0 & 0 & 0 & $4 / 4 / 6$ & 0 \\
\hline 4 & 61 & 8 & 1 & L/B & 1 & 1 & 0 & 0 & D/Sorafenib & $6 / 6 / 6$ & Liver \\
\hline 5 & 67 & 9 & 0 & L/B & 1 & 0 & 0 & 0 & 0 & $6 / 6 / 6$ & 0 \\
\hline 6 & 78 & 8 & 1 & L/B & 1 & 0 & 0 & 0 & 0 & $6 / 6 / 6$ & 0 \\
\hline 7 & 71 & 9 & 0 & 0 & 1 & 0 & 1 & 0 & D/C/EMP/HU & 4/PD & Liver \\
\hline 8 & 78 & $7 b$ & 1 & B & 1 & 1 & 1 & 1 & D/EMP & 6 & 0 \\
\hline 9 & 68 & 9 & 1 & 0 & 1 & 0 & 0 & 0 & $\mathrm{D}$ & $6 / 6 / 6$ & Brain \\
\hline 10 & 74 & 9 & 1 & 0 & 1 & 1 & 1 & 0 & 0 & $4 / 6 / 6$ & Liver \\
\hline 11 & 66 & 9 & 1 & $\mathrm{~L}$ & 1 & 1 & 0 & 0 & 0 & $6 / 6 / 6$ & 0 \\
\hline 12 & 78 & 8 & 1 & 0 & 1 & 0 & 0 & 0 & 0 & $6 / 6$ & 0 \\
\hline 13 & 79 & $7 b$ & 1 & 0 & 1 & 0 & 0 & 1 & 0 & 3/Tox & Lung, adrenal \\
\hline 14 & 73 & 9 & 1 & B & 1 & 1 & 1 & 0 & 0 & $4 / 6 / 6$ & Liver, adrenal \\
\hline 15 & 71 & 7 & 0 & $L$ & 1 & 1 & 0 & 0 & 0 & $4 / 6$ & Liver \\
\hline 16 & 68 & NA & 0 & 0 & 1 & 1 & 0 & 1 & D/EMP & 6 & 0 \\
\hline 17 & 73 & NA & 1 & L/B & 1 & 1 & 0 & 1 & 0 & $4 / 4$ & 0 \\
\hline 18 & 78 & 8 & 1 & $L$ & 1 & 1 & 0 & 1 & 0 & $4 / 6 / 6$ & 0 \\
\hline 19 & 73 & NA & 1 & L/B & 1 & 1 & 0 & 0 & $\mathrm{D}$ & 4/Tox & Lung \\
\hline 20 & 68 & 7 & 1 & B & 1 & 1 & 1 & 0 & $\mathrm{D}$ & 6 & 0 \\
\hline 21 & 85 & $7 a$ & 1 & $B$ & 1 & 1 & 1 & 0 & D & $6 / 6 /$ & 0 \\
\hline 22 & 71 & 7 & 0 & $L$ & 1 & 1 & 0 & 0 & 0 & 4/PD & Rectum \\
\hline 23 & 66 & 9 & 1 & L/B & 1 & 1 & 1 & 0 & 0 & $6 / 6$ & 0 \\
\hline 24 & 75 & 8 & 1 & B & 1 & 1 & 1 & 0 & $\mathrm{D}$ & 6 & 0 \\
\hline 25 & 80 & 7 & 1 & B & 1 & 1 & 1 & 0 & $\mathrm{D} / \mathrm{C}$ & 6 & Liver, lung \\
\hline 26 & 64 & 9 & 0 & B & 1 & 1 & 0 & 1 & 0 & 6 & 0 \\
\hline 27 & 61 & 9 & 1 & L/B & 1 & 1 & 1 & 0 & $\mathrm{D} / \mathrm{C}$ & 6 & Liver \\
\hline 28 & 69 & 8 & 1 & L/B & 1 & 1 & 0 & 0 & 0 & $6 / 6 /$ & Lung \\
\hline 29 & 73 & 9 & 0 & $L$ & 1 & 1 & 1 & 0 & D & $6 / 6$ & 0 \\
\hline 30 & 75 & NA & 1 & $L$ & 1 & 0 & 1 & 0 & 0 & 6/Tox & 0 \\
\hline
\end{tabular}

GS = Gleason score; OP = prostatectomy; RTx = radiation therapy to prostate bed $(=\mathrm{L})$ or bone $(=\mathrm{B})$; CRPC = hormone therapy with both a luteinizing hormone-releasing hormone (LHRH) analog/antagonist and an antiandrogen; Abirat = abiraterone; Enza = enzalutamide; CTx = chemotherapy with docetaxel (=D), cabazitaxel $(=\mathrm{C})$, estramustine monophosphate (= EMP), or hydroxyurea $(=\mathrm{HU})$; Cycles = therapy with ${ }^{177}$ Lu-PSMA-617 with given activities (GBq) in bimonthly fractions; NA = not available.

Fractionated therapy had to be discontinued due to toxicity (= Tox) or progressive disease (= PD).

(including tumor tissue) assuming equilibrium between blood and the particular compartment, respectively. Approximately 50\% of the injected activity was eliminated by urine during the first $48 \mathrm{~h}$, then the cumulative clearance curve reached a plateau (Supplemental Fig. 1B). The intestine presented maximum contrast in the 20-h postinjection image, followed by a normal colon passage speed. Approximately $1 \%-5 \%$ of the injected dose was eliminated by fecal excretion.

After $48 \mathrm{~h}$, the direct $\gamma$-emission was less than $2 \mu \mathrm{Sv} / \mathrm{h}$ at $2 \mathrm{~m}$ distance for all patients. Because of the observation that urine clearance of non-tumor-bound PSMA-617 is almost completed $48 \mathrm{~h}$ after injection and clearance from the intestine can be stimulated with moderate laxatives administered $24 \mathrm{~h}$ after ${ }^{177}$ Lu-PSMA-617, all patients could be discharged after $48 \mathrm{~h}$ in accordance with our currently valid radiation protection regulations (18).

\section{Dosimetry}

The dosimetry analyses of 4 patients during their first and second treatment cycles revealed a mean $( \pm \mathrm{SD})$ kidney dose of $0.75 \pm$ $0.19 \mathrm{~Gy} / \mathrm{GBq}$ of ${ }^{177} \mathrm{Lu}-\mathrm{PSMA}-617$. The red marrow dose was $0.03 \pm$ 0.01 , parotid $1.28 \pm 0.40$, and submandibular gland $1.48 \pm 0.37 \mathrm{~Gy} /$ $\mathrm{GBq}$. There was no relevant difference in dosimetry for the patients 
TABLE 2

Dosimetry

\begin{tabular}{|c|c|c|c|c|c|c|c|c|}
\hline \multirow[b]{3}{*}{ Tumor load } & \multicolumn{8}{|c|}{ Patient-cycle } \\
\hline & \multicolumn{2}{|c|}{ Low } & \multicolumn{2}{|c|}{ Intermediate-low } & \multicolumn{2}{|c|}{ Intermediate-high } & \multicolumn{2}{|c|}{ High } \\
\hline & $\begin{array}{c}\mathrm{P} 1-\mathrm{C} 1 \\
(\mathrm{~Gy} / \mathrm{GBq})\end{array}$ & $\begin{array}{c}\mathrm{P} 1-\mathrm{C} 2 \\
\text { (Gy/GBq) }\end{array}$ & $\begin{array}{c}\mathrm{P} 2-\mathrm{C} 1 \\
\text { (Gy/GBq) }\end{array}$ & $\begin{array}{c}\text { P2-C2 } \\
\text { (Gy/GBq) }\end{array}$ & $\begin{array}{c}\text { P3-C1 } \\
\text { (Gy/GBq) }\end{array}$ & $\begin{array}{c}\text { P3-C2 } \\
\text { (Gy/GBq) }\end{array}$ & $\begin{array}{c}\text { P4-C1 } \\
\text { (Gy/GBq) }\end{array}$ & $\begin{array}{c}\text { P4-C2 } \\
\text { (Gy/GBq) }\end{array}$ \\
\hline Kidney & 0.55 & 0.56 & 1.14 & 0.82 & 0.81 & 0.76 & 0.62 & 0.76 \\
\hline Red marrow & 0.02 & 0.02 & 0.02 & 0.02 & 0.03 & 0.03 & 0.05 & 0.03 \\
\hline Parotid gland & 2.2 & 1.16 & 1.03 & 0.82 & 1.26 & 1.3 & 1.27 & 1.17 \\
\hline Submandibular gland & 1.3 & 1.69 & 1.26 & 0.97 & 1.37 & 1.31 & 1.82 & 2.13 \\
\hline Liver & 0.09 & 0.1 & 0.07 & 0.06 & 0.09 & 0.1 & 0.16 & 0.13 \\
\hline Spleen & 0.19 & 0.15 & 0.26 & 0.14 & 0.11 & 0.13 & 0.28 & 0.27 \\
\hline Bladder wall & 0.03 & 0.16 & 0.16 & 0.17 & 0.29 & 0.23 & 0.41 & 0.36 \\
\hline Metastases (mean) & 6.1 & & 22.8 & & 15.3 & & 14 & \\
\hline Effective dose equivalent (mSv/GBq) & 81.8 & 77 & 114 & 82.5 & 96.3 & 91.2 & 126 & 111 \\
\hline Effective dose (mSv/GBq) & 48.2 & 46.5 & 37.2 & 43.9 & 58.3 & 54.3 & 83,4 & 65 \\
\hline
\end{tabular}

with low or high tumor load. In addition, there was no relevant difference in the kidney and red marrow dose between the first and second treatment cycle. Distinct values and additional (not doselimiting) organs are presented in Table 2. The red marrow dose consists of an approximately $45 \%$ self-dose-that is, $\beta$-radiation during perfusion and passive diffusion into the interstitial spaceand $55 \%$ spill-in radiation (5\% from the delineable source organs, $50 \%$ from the remainder body including tumor lesions).

\section{Treatment Efficacy}

Eight weeks after the first treatment cycle, 21 of 30 patients demonstrated a decrease in PSA; in 18 patients the decrease was more than $25 \%$ and in 13 patients more than $50 \%$. However, 8 patients demonstrated a rising PSA and 1 patient remained stable (Fig. 2A). After $24 \mathrm{wk}$, that is, nearly 6 mo after initial therapy, 9 of 11 patients receiving 3 treatment cycles presented with a sustained decrease in PSA in comparison to the baseline value; the decrease was more than $25 \%$ for all of these 9 patients and more than $50 \%$ in 8 patients (Fig. 2B). Follow-up between the week- 8 and week-24 PSA response (Fig. 2C) revealed that in 8 of 11 patients the PSA levels further decreased from cycle 1 to cycle 3 . One patient who already presented with PSA progression after the first cycle continued therapy because of favorable symptomatic response and had further PSA progression after the third cycle. Two patients initially responded to cycle 1 but had PSA relapse by cycle 3; however, in one of these patients the PSA was still less than 50\% in comparison to baseline. In these patients, imaging findings also demonstrated partial remission in comparison to baseline staging.

Imaging-based restaging revealed a positive response in 10 of the 11 patients; surprisingly, a positive imaging response was even found in 1 of the 2 patients with rising PSA. Six patients were restaged with PSMA PET/CT, and all presented with a decrease of more than $50 \%$ (average of index lesions) in $\mathrm{SUV}_{\max }$ (Fig. 3A). Three patients were assessed with ${ }^{99 \mathrm{~m} T c-P S M A}$ SPECT/CT and presented with visual response (Fig. 3B). In patients with softtissue or lymph node metastases (target lesions according to RECIST), response was additionally demonstrated with CT (Fig. $3 \mathrm{C})$. Also, the posttherapeutic emission scans based on the inherent imaging capabilities of ${ }^{177} \mathrm{Lu}$ (coemission of $\gamma$-radiation) seem sufficient to monitor treatment response despite a minimal lower resolution and higher noise (Fig. 4). Because of the multitude of lesions, we did not assess the exact lesion number; as long as the total number of delineable metastases decreased by visual estimation, the situation was considered a radiologic response. Thus, similar to the use of bone scans in clinical trials (19), single new lesions were not considered progressive disease.

Clinically, the treatment was able to stabilize the patient's well-being. None of the patients discontinued treatment because of a worsening of their general clinical condition. The body weight remained fairly stable (mean body weight at baseline, $83 \mathrm{~kg}$; at week $24,81 \mathrm{~kg}$ ). None of the 24 of 30 patients without opioid analgesics at baseline had to start such a medication during followup. The dose of the 6 of 30 patients with opioid analgesics at baseline remained stable.

\section{Treatment Toxicity}

Creatinine and urea as well as liver enzymes were not significantly changed during the complete follow-up period, which was $12 \mathrm{wk}$ for the 19 patients receiving 1 treatment cycle and $24 \mathrm{wk}$ for the 11 patients receiving 3
FIGURE 2. (A) Waterfall graph presenting PSA response after 1 cycle of ${ }^{177}$ Lu-PSMA-617 therapy. (B) Waterfall graph presenting PSA response after 3 cycles of therapy. (C) Follow-up between PSA response after cycle 1 and cycle 3.

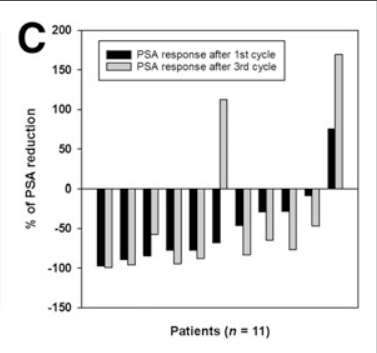




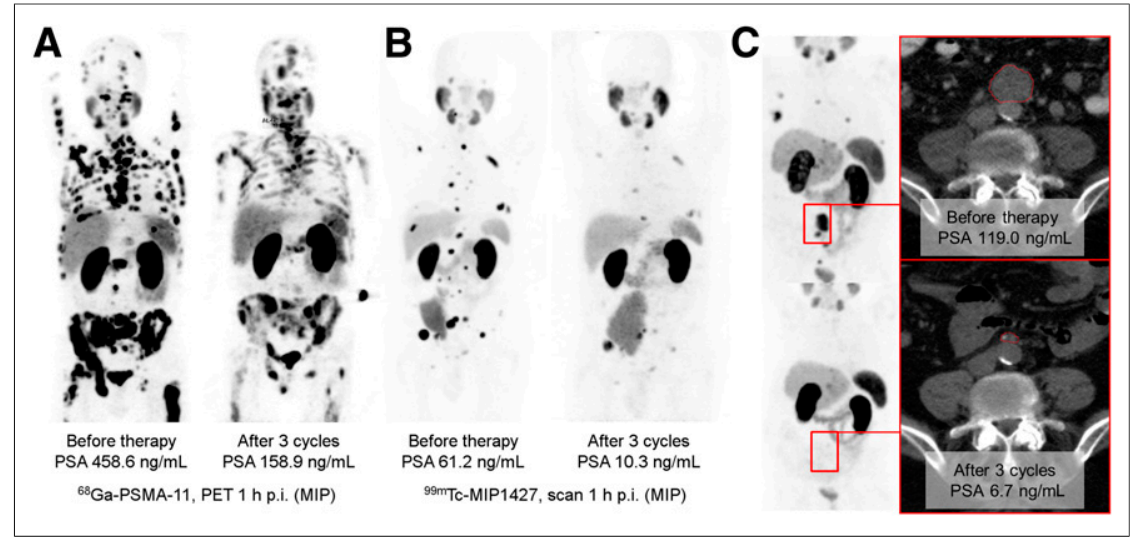

FIGURE 3. Imaging-based response evaluation. (A) ${ }^{68}$ Ga-PSMA-11 PET was evaluated semiquantitatively. (B) ${ }^{99 m}$ Tc-MIP1427 scintigraphy-enabled visual evaluation. (C) If target lesions were available, CT was evaluated in accordance to RECIST. GM = geometric mean; MIP = maximum-intensity projection; p.i. $=$ after injection.

treatment cycles. Thus, follow-up was sufficient to report acute and midterm toxicities but not late effects.

Among 15 patients with normal baseline hemoglobin, 6 patients developed grade I anemia, and 9 had no red cell toxicity. In 10 patients with grade I anemia before therapy, only 3 patients had a decline to grade II, 6 patients remained stable, and 1 patient improved to the reference range (this patient simultaneously presented with striking radiologic improvement of bone metastases). For 3 patients who already had grade II anemia at baseline, 1 worsened to grade III (after only 1 treatment cycle), 1 was stable, and 1 improved to grade I. In comparison to baseline, 18 of 27 patients had no worsening of anemia (66\%) and 9 worsened by 1 grade (33\%); no patient had a decline of more than 1 grade. The only patient with grade III anemia had diffusepattern bone marrow involvement on pretherapeutic imaging. Two patients had already received substitution of erythrocytes less than $6 \mathrm{wk}$ before PSMA therapy and were omitted from evaluation of anemia.

With regard to white blood cell count (Fig. 5A), 22 patients never developed Common Terminology Criteria for Adverse Events toxicity higher than baseline. Grade I leucopenia was observed in 6 infrequently.

\section{DISCUSSION} moderate toxicities. patients mainly after the third cycle. Grade II was observed in 2 patients, both with diffuse-pattern bone marrow involvement.

Platelet count (Fig. 5B) demonstrated high interindividual variability. However, in 23 patients the absolute platelet count never dropped below the reference range. In 4 patients, grade I thrombocytopenia was observed. One patient developed grade II and 1 patient grade III thrombocytopenia. Both patients had previously presented with diffuse-pattern bone marrow infiltration during imaging and were the same patients who developed the highest white blood cell toxicity. In 1 patient, grade IV thrombocytopenia was already present at baseline. Despite the fact that the absolute platelet count stayed within the reference range $(150-300 / \mathrm{nL})$ for 23 of 30 patients, we observed a relative decline in the mean platelet count of $-14 \%$, with nadir 4-6 wk after the first therapy that recovered after $8 \mathrm{wk}$. However, in the 11 patients receiving 3 cycles we found a chronic decrease of platelets $(-20 \%)$ from baseline to week 24 .

Most of the patients reported no relevant dysfunction of salivary glands. Substitution of saliva (spray/gel) was prescribed to 2 of 30 patients; both developed xerostomia after the third cycle. After the first and second treatment cycle only temporal xerostomia without relevant loss in quality of life was occasionally reported. Mild fatigue over baseline was regularly reported, but only 2 times was it attributed to affecting activities of daily living. Nausea and loss of appetite during the first weeks after therapy were reported

Here, we report our clinical experience with ${ }^{177}$ Lu-PSMA-617, which revealed antitumor activity in most patients with mild to

In contrast to conventional pharmaceuticals, the toxicity and response probability of a radiopharmaceutical predominantly depends on the radiation absorbed dose to healthy and tumor tissue, respectively. There are welldefined radiation tolerance limits for normal organs. Therefore, empiric dose escalation studies can partially be omitted and dosing of radioactive drugs can be based on dosimetry. Our dosimetry data are well in line with 2 other recent investigations (20,21). The highest normal-organ dose was found for the salivary glands. Thus, the sporadic incidence of reversible xerostomia, which was mainly observed after the third cycle, is reasonable, taking into account published radiation tolerance limits (22). However, if mild xerostomia is considered to be an annoying but harmless side effect, kidneys are the only essential dose-limiting organs and their tolerance limits would permit about twice the cumulative 

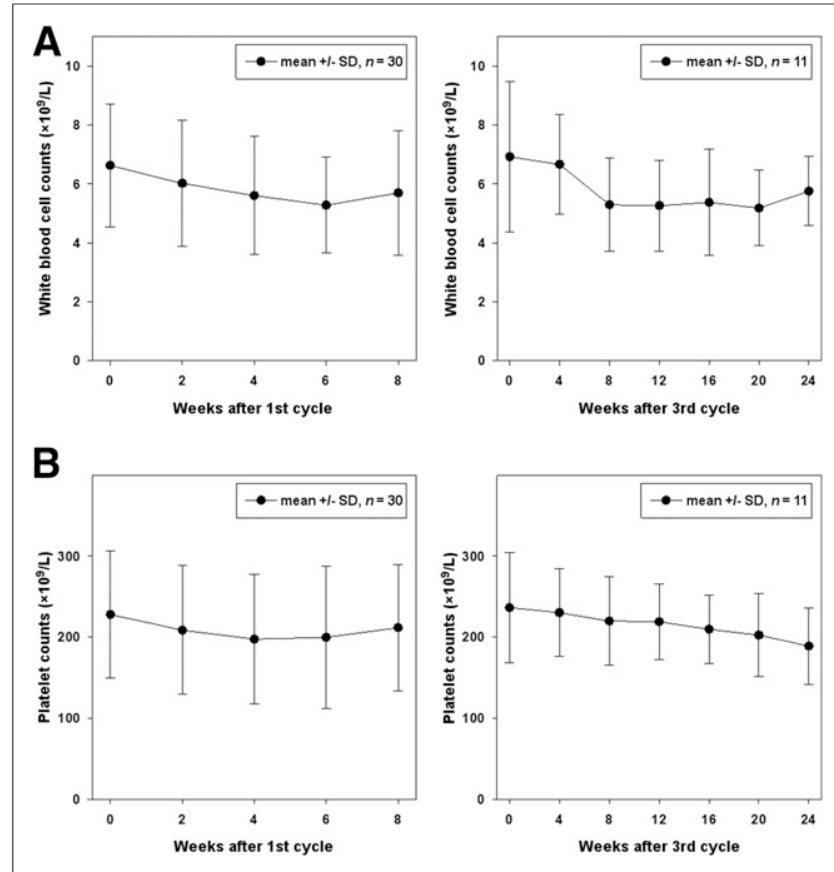

FIGURE 5. Course of white blood cell count (A) and platelets (B) during ${ }^{177}$ Lu-PSMA-617 therapy.

dose-that is, $36 \mathrm{GBq}$ of ${ }^{177} \mathrm{Lu}-\mathrm{PSMA}-617$ (23)—which conversely would still stay below the limits to provoke severe and irreversible xerostomia (22). Additionally, recent attempts to reduce kidney uptake of PSMA ligands raise hope to further increase the therapeutic index (24). Selecting the ideal single fraction dose is more challenging because bone marrow reserve can be reduced after previous chemotherapy, and the published tolerance limits are not reliable (25). Also dosimetry can underestimate red marrow dose because the $\beta$-radiation arising from bone metastases cannot be sufficiently modeled. The $497-\mathrm{keV} \beta$-energy of ${ }^{177} \mathrm{Lu}$ corresponds to a mean and maximum tissue range of only 0.5 and $2 \mathrm{~mm}$ (i.e., 10-50 cell diameters), respectively, and it is plausible to neglect this dose contribution if only a limited number of solid bone metastases are present. However, it might be relevant in the case of diffuse bone marrow involvement. Therefore, we initially administered conservative 4-GBq fractions. Once it became clear that only diffuse-type bone marrow involvement, eventually in combination with previous chemotherapy, presented a risk factor for higher hematotoxicity, we escalated to $6 \mathrm{GBq}$ and patients with diffuse-pattern bone marrow involvement were subsequently stratified to receive PSMA-617 labeled with an $\alpha$-emitter. Targeted $\alpha$-radiation therapy was already demonstrated to reduce red marrow toxicity in similar situations (26). However, the reliability of this tailored approach has still to be proven. Despite moderate acute hematotoxicity, we observed a chronic decline of platelets during 3 cycles, thus further dose escalations of ${ }^{177} \mathrm{Lu}$-PSMA-617 should be conducted cautiously. Nevertheless, there is still some room to improve the treatment regime.

The main limitation of this report is that the patients were not systematically selected in a prospective manner with stringent inclusion criteria such as in a typical clinical trial. Therefore, the results of this retrospective evaluation should be considered only explorative. Nevertheless, the findings are noteworthy in view of the high number of prior treatments seen by our patients before receiving ${ }^{177} \mathrm{Lu}$-PSMA-617. The novel $\mathrm{mCRPC}$ agents have been approved with hormone therapy (Cougar-302, PREVAIL) or hormone and docetaxel (Cougar-301, AFFIRM, TROPIC) being the only pretreatments (27). In contrast, if the novel drugs are applied consecutively, the more than 50\% PSA response rate is commonly less than $40 \%$ (28). Our cohort is high risk, with negative prognostic factors such as high Gleason score and visceral metastases (29), making the high response rate with the absence of severe toxicity all the more remarkable.

It has been reported that tubulin-targeting with taxanes inhibits androgen receptor nuclear translocation (30). Because abiraterone or enzalutamide also interfere with androgen receptor signaling, these drugs are somehow competitive in their mechanism of action and cross resistance may occur, making optimal sequencing of the new drugs challenging $(28,30)$. In contrast, PSMA genes are suppressed by androgens, and androgen independency as well as androgen-deprivation therapy may even increase the expression of PSMA in mCRPC $(31,32)$. Thus, PSMA targeting is rather complementary to the currently approved drugs and can still be effective when targeting the androgen receptor axis fails. This would explain the high rate of radiologic and PSA responses despite excessive pretreatment.

On the other hand, the reported patients include some selection bias. Patients with diffuse bone marrow involvement were excluded, once it became apparent that these patients have a higher probability to develop hematotoxicity. Additionally, a PSMA-positive tumor phenotype based on PET or scintigraphy was a precondition to receive therapy. However, treatment stratification based on prognostic factors is a desired objective in modern oncology, and it is beneficial that PSMA-positive tumors can be easily identified noninvasively with PSMA imaging (33). In addition, a diagnostic study with PSMA PET/CT found PSMA-positive tumor phenotypes in $88 \%$ of prostate cancer relapses, suggesting that most mCRPC patients may be potential candidates for PSMA-targeted therapy (34).

\section{CONCLUSION}

${ }^{177} \mathrm{Lu}$-PSMA-617 is a new treatment option for mCRPC that demonstrates substantial antitumor activity with few side effects. ${ }^{177}$ Lu-PSMA-617, therefore, deserves more attention in larger prospective trials.

\section{DISCLOSURE}

The costs of publication of this article were defrayed in part by the payment of page charges. Therefore, and solely to indicate this fact, this article is hereby marked "advertisement" in accordance with 18 USC section 1734. This research was supported by the Klaus-Tschira-Stiftung (project no. 00.198.2012). There is a patent pending for PSMA-617 (Martina Benešová, Matthias Eder, Klaus Kopka, and Uwe Haberkorn). No other potential conflict of interest relevant to this article was reported.

\section{REFERENCES}

1. Bostwick DG, Pacelli A, Blute M, Roche P, Murphy GP. Prostate specific membrane antigen expression in prostatic intraepithelial neoplasia and adenocarcinoma: a study of 184 cases. Cancer. 1998;82:2256-2261.

2. Perner S, Hofer MD, Kim R, et al. Prostate-specific membrane antigen expression as a predictor of prostate cancer progression. Hum Pathol. 2007;38:696-701.

3. Liu H, Rajasekaran AK, Moy P, et al. Constitutive and antibody-induced internalization of prostate-specific membrane antigen. Cancer Res. 1998;58:4055-4060. 
4. Ma D, Hopf CE, Malewicz AD, et al. Potent antitumor activity of an auristatinconjugated, fully human monoclonal antibody to prostate-specific membrane antigen. Clin Cancer Res. 2006;12:2591-2596.

5. Rose JN, Crook JM. The role of radiation therapy in the treatment of metastatic castrate-resistant prostate cancer. Ther Adv Urol. 2015;7:135-145.

6. Tagawa ST, Milowsky MI, Morris M, et al. Phase II study of lutetium-177labeled anti-prostate-specific membrane antigen monoclonal antibody J591 for metastatic castration-resistant prostate cancer. Clin Cancer Res. 2013;19:51825191.

7. Vallabhajosula S, Goldsmith SJ, Hamacher KA, et al. Prediction of myelotoxicity based on bone marrow radiation-absorbed dose: radioimmunotherapy studies using ${ }^{90} \mathrm{Y}$ - and ${ }^{177} \mathrm{Lu}$-Labeled J591 antibodies specific for prostate-specific membrane antigen. J Nucl Med. 2005;46:850-858.

8. Vallabhajosula S, Goldsmith SJ, Kostakoglu L, Milowsky MI, Nanus DM, Bander NH. Radioimmunotherapy of prostate cancer using ${ }^{90} \mathrm{Y}$ - and ${ }^{177} \mathrm{Lu}$-labeled J591 monoclonal antibodies: effect of multiple treatments on myelotoxicity. Clin Cancer Res. 2005;11:7195s-7200s.

9. Zechmann CM, Afshar-Oromieh A, Armor T, et al. Radiation dosimetry and first therapy results with a ${ }^{124} \mathrm{I} /{ }^{131} \mathrm{I}$-labeled small molecule (MIP-1095) targeting PSMA for prostate cancer therapy. Eur J Nucl Med Mol Imaging. 2014;41:1280-1292.

10. Benešová M, Schäfer M, Bauder-Wüst U, et al. Preclinical evaluation of a tailormade DOTA-conjugated PSMA inhibitor with optimized linker moiety for imaging and endoradiotherapy of prostate cancer. J Nucl Med. 2015;56:914-920.

11. Kratochwil C, Giesel FL, Eder M, et al. [ ${ }^{177}$ Lu]lutetium-labelled PSMA ligandinduced remission in a patient with metastatic prostate cancer. Eur J Nucl Med Mol Imaging. 2015;42:987-988.

12. Gourni E, Canovas C, Goncalves V, Denat F, Meyer PT, Maecke HR. (R)-NODAGAPSMA: A versatile precursor for radiometal labeling and nuclear imaging of PSMApositive tumors. PLoS One. 2015;10:e145755.

13. Barinka C, Byun Y, Dusich CL, et al. Interactions between human glutamate carboxypeptidase II and urea-based inhibitors: structural characterization. J Med Chem. 2008;51:7737-7743.

14. Lu G, Maresca KP, Hillier SM, et al. Synthesis and SAR of ${ }^{99 \mathrm{~m}} \mathrm{Tc} / \mathrm{Re}$-labeled small molecule prostate specific membrane antigen inhibitors with novel polar chelates. Bioorg Med Chem Lett. 2013;23:1557-1563.

15. Eder M, Neels O, Müller M, et al. Novel preclinical and radiopharmaceutical aspects of $\left[{ }^{68} \mathrm{Ga}\right] \mathrm{Ga}-\mathrm{PSMA}-\mathrm{HBED}-\mathrm{CC}$ : a new PET tracer for imaging of prostate cancer. Pharmaceuticals (Basel). 2014;7:779-796.

16. Common Terminology Criteria for Adverse Events v3.0 (CTCAE). cancer.gov website. http://ctep.cancer.gov/protocolDevelopment/electronic_applications/docs/ ctcaev3.pdf. Published August 9, 2006. Accessed April 14, 2016.

17. Leard SE, Freis ED. Changes in the volume of the plasma, interstitial and intracellular fluid spaces during hydration and dehydration in normal and edematous subjects. Am J Med. 1949;7:647-654.

18. SSK. Notwendigkeit der stationären Durchführung der Ganzkörperszintigraphie mit I-131 beim Schilddrüsenkarzinom. Strahlenschutzkommission website. http://www.ssk.de/SharedDocs/Beratungsergebnisse_PDF/2004/Ganzkoerperszintigraphie_I131.html?nn=2241514. BAnz Nr. 158. Published April 22, 2004. Accessed April 14, 2016.
19. Scher HI, Halabi S, Tannock I, et al. Design and end points of clinical trials for patients with progressive prostate cancer and castrate levels of testosterone: recommendations of the Prostate Cancer Clinical Trials Working Group. J Clin Oncol. 2008;26:1148-1159.

20. Delker A, Fendler WP, Kratochwil C, et al. Dosimetry for ${ }^{177}$ Lu-DKFZ-PSMA617: a new radiopharmaceutical for the treatment of metastatic prostate cancer. Eur J Nucl Med Mol Imaging. 2016;43:42-51.

21. Kabasakal L, AbuQbeitah M, Aygün A, et al. Pre-therapeutic dosimetry of normal organs and tissues of ${ }^{177}$ Lu-PSMA-617 prostate-specific membrane antigen (PSMA) inhibitor in patients with castration-resistant prostate cancer. Eur J Nucl Med Mol Imaging. 2015;42:1976-1983.

22. Hey J, Setz J, Gerlach R, et al. Parotid gland-recovery after radiotherapy in the head and neck region: 36 months follow-up of a prospective clinical study. Radiat Oncol. 2011;6:125.

23. Cremonesi M, Ferrari M, Di Dia A, et al. Recent issues on dosimetry and radiobiology for peptide receptor radionuclide therapy. Q J Nucl Med Mol Imaging. 2011;55:155-167.

24. Kratochwil C, Giesel FL, Leotta K, et al. PMPA for nephroprotection in PSMAtargeted radionuclide therapy of prostate cancer. J Nucl Med. 2015;56:293-298.

25. Siegel JA, Yeldell D, Goldenberg DM, et al. Red marrow radiation dose adjustment using plasma FLT3-L cytokine levels: improved correlations between hematologic toxicity and bone marrow dose for radioimmunotherapy patients. J Nucl Med. 2003;44:67-76.

26. Kratochwil C, Giesel FL, Bruchertseifer F, et al. ${ }^{213}$ Bi-DOTATOC receptortargeted alpha-radionuclide therapy induces remission in neuroendocrine tumours refractory to beta radiation: a first-in-human experience. Eur J $\mathrm{Nucl} \mathrm{Med} \mathrm{Mol}$ Imaging. 2014;41:2106-2119.

27. Crawford ED, Higano CS, Shore ND, Hussain M, Petrylak DP. Treating patients with metastatic castration resistant prostate cancer: a comprehensive review of available therapies. J Urol. 2015;194:1537-1547.

28. Chi K, Hotte SJ, Joshua AM, et al. Treatment of mCRPC in the AR-axis-targeted therapy-resistant state. Ann Oncol. 2015;26:2044-2056.

29. Pond GR, Sonpavde G, de Wit R, Eisenberger MA, Tannock IF, Armstrong AJ. The prognostic importance of metastatic site in men with metastatic castrationresistant prostate cancer. Eur Urol. 2014;65:3-6.

30. van Soest RJ, van Royen ME, de Morrée ES, et al. Cross-resistance between taxanes and new hormonal agents abiraterone and enzalutamide may affect drug sequence choices in metastatic castration-resistant prostate cancer. Eur J Cancer. 2013;49:3821-3830.

31. Evans MJ, Smith-Jones PM, Wongvipat J, et al. Noninvasive measurement of androgen receptor signaling with a positron-emitting radiopharmaceutical that targets prostate-specific membrane antigen. Proc Natl Acad Sci USA. 2011;108: 9578-9582.

32. Wright GL Jr, Grob BM, Haley C, et al. Upregulation of prostate-specific membrane antigen after androgen-deprivation therapy. Urology. 1996;48:326-334.

33. Lee DY, Li KC. Molecular theranostics: a primer for the imaging professional. AJR. 2011;197:318-324.

34. Afshar-Oromieh A, Avtzi E, Giesel FL, et al. The diagnostic value of PET/CT imaging with the ${ }^{68} \mathrm{Ga}$-labelled PSMA ligand HBED-CC in the diagnosis of recurrent prostate cancer. Eur J Nucl Med Mol Imaging. 2015;42:197-209. 